\title{
A way of decoupling gravitational sources in pure Lovelock gravity
}

\author{
Milko Estrada ${ }^{\mathrm{a}}$ \\ Departamento de Física, Facultad de ciencias básicas, Universidad de Antofagasta, Casilla 170, Antofagasta, Chile
}

Received: 26 July 2019 / Accepted: 30 October 2019 / Published online: 12 November 2019

(c) The Author(s) 2019

\begin{abstract}
We provide an algorithm that shows how to decouple gravitational sources in pure Lovelock gravity. This method allows to obtain several new and known analytic solutions of physical interest in scenarios with extra dimensions and with presence of higher curvature terms. Furthermore, using our method, it is shown that applying the minimal geometric deformation to the Anti de Sitter space time it is possible to obtain regular black hole solutions.
\end{abstract}

\section{Introduction}

In the last years, several branches of theoretical physics have predicted the presence of extra dimensions. Thus, it makes sense to think of the existence of geometrical objects in space times with a number of dimensions greater than four as for example branes, strings, or higher dimensional black holes. In regarding this, theories of gravity emerged which present higher curvature correction terms when the space time has a number of dimensions greater than four. One interesting theory among of them is Lovelock gravity [1]. One achievement of Lovelock theory is that it shares the following features with the general relativity.

- Its equation of motion are symmetric.

- Its equations of motion are of second order on the derivative of the metric tensor.

- Free divergence.

Into the Lovelock gravities, we can find the Pure Lovelock theory. It is well known that the general relativity has a no non-trivial vacuum solution (without cosmological constant) when $d=3$ (i.e $d=2 n+1$, where $n=1$ ), one interesting feature is that Pure Lovelock keeps this property for $d=$ $2 n+1$ with $n>1$, see Ref. [2]. On the other hand, including the cosmological constant, general relativity has a unique

a e-mail: milko.estrada@ua.cl
(Anti) de Sitter ground state for $\Lambda(<0)>0$, in regarding this, other interesting feature of Pure Lovelock theory is that, it keeps this feature for $n$ odd, however, for $n$ even, this theory has a double Anti de Sitter or de Sitter ground state for $\Lambda>0$ and does not have ground state for $\Lambda<0[3,4]$.

On the other hand, in Einstein Hilbert theory, finding new solutions of physical interest is not a simple task due to the highly nonlinear behavior of its equations. In regarding this, Ovalle [5] proposed a method called Gravitational Decoupling of Sources, which corresponds to the first algorithm that shows how to decouple gravitational sources in General Relativity. This method applies a Minimal Geometric Deformation (MGD) to the temporal and radial metric components together with a decoupling of sources. The method is explained in Ref. [6]: "given two gravitational sources: a source A and an extra source B, standard Einstein's equations are first solved for $\mathrm{A}$, and then a simpler set of quasi-Einstein equations are solved for B. Finally, the two solutions can be combined in order to derive the complete solution for the total system.".

By applying this method to a solution of Einstein equations, named seed solution, it is possible to obtain new analytic solutions of physical interest. Related to this, by deforming some isotropic well known and well behaved solutions, new anisotropic and well behaved solutions have been obtained that represent stellar distributions in Refs. [6-13]. By deforming the Schwarzschild space time new black hole solutions have been obtained in Ref. [14] (other black hole solutions obtained with this method in Refs. [15-18]). Other examples of applications of the method are: solutions in Einstein Klein Gordon system [19]; solutions in $f(G)$ gravity [20]; solutions in $f(R)$ gravity [21], cloud of strings solutions [22]. See other applications in Refs. [23-27,27-31].

Thus, motivated by the fact that in Pure Lovelock theory there are several kind of solutions in literature as for example: black hole solutions in Refs. [4,32-36] and stellar distributions in Refs. [37-39], it seems of physical interest to provide an algorithm to decouple gravitational sources in Pure Love- 
lock gravity, and thus, to apply the method to known ( or unknown) solutions and to test if it is possible to obtain new solutions of physical interest. See also $[3,40,41]$.

We start by deforming the seed energy momentum tensor $\bar{T}_{A B}$ by an additional source $\theta_{A B}$, which causes anisotropic effects on the self-gravitating system. This additional source can contain new fields, like scalar, vector and tensor fields [6]. Therefore the energy momentum tensor is:

$T_{A B}=\bar{T}_{A B}+\theta_{A B}$,

since Pure Lovelock theory has free divergence, then, the energy momentum tensor satisfies the conservation equation:

$\nabla_{A} T^{A B}=0$.

In this work we provide a Gravitational Decoupling method in Pure Lovelock gravity, and therefore we show a simple approach to decoupling gravitational sources in this theory. We will show that the Pure Lovelock equations of motion can be solved for each component $\left\{\bar{T}_{A B}, \theta_{A B}\right\}$ separately, at least for the spherically symmetric and static case. For each component will be obtained a particular metric tensor $\left\{\bar{g}_{A B}, g_{A B}^{\theta}\right\}$, and the final metric $g_{A B}$ is a simple combination of these metrics. As a simple test, we will apply our method to an Anti de Sitter space time and, we will test if it is possible to obtain solutions that represent regular black holes.

\section{Lovelock Gravity and the Pure Lovelock case}

The Lovelock Lagrangian is :

$L=\sqrt{-g} \sum_{n=0}^{N} \gamma_{n} L_{n}$,

where $N=\frac{d}{2}-1$ for $d$ even and $N=\frac{d-1}{2}$ for $d$ odd and, $\gamma_{n}$ are arbitrary coupling constants. $L_{n}$ is a topological density defined as:

$L_{n}=\frac{1}{2^{n}} \delta_{\alpha_{1} \beta_{1} \cdots \alpha_{n} \beta_{n}}^{\mu_{1} \nu_{1} \cdots \mu_{n} v_{n}} \Pi_{r=1}^{n} R_{\mu_{r} v_{r}}^{\alpha_{r} \beta_{r}}$,

where $R_{\mu \nu}^{\alpha \beta}$ is a $n$ order generalization of the Riemann tensor for the Lovelock theory, and:

$\delta_{\alpha_{1} \beta_{1} \cdots \alpha_{n} \beta_{n}}^{\mu_{1} \nu_{1} \cdots \mu_{n} v_{n}}=\frac{1}{n !} \delta_{\left[\alpha_{1}\right.}^{\mu_{1}} \delta_{\beta_{1}}^{\nu_{1}} \cdots \delta_{\alpha_{n}}^{\mu_{n}} \delta_{\left.\beta_{n}\right]}^{v_{n}}$

is the generalized Kronecker delta.

It is worth to stress that, the terms $L_{0}, L_{1}$ and $L_{2}$ are proportional to the cosmological constant, Ricci Scalar and the Gauss Bonnet Lagrangian, respectively. The corresponding equation of motion is given by:
$\sum_{n=0}^{N} \gamma_{n} \mathcal{G}_{A B}^{(n)}=T_{A B}$

where $\mathcal{G}_{A B}^{(n)}$ is a $n$ order generalization of the Einstein tensor due to the topological density $L_{n}$. As example $\mathcal{G}_{A B}^{(1)}$ is just the Einstein tensor associated with the Ricci scalar (Einstein Hilbert theory is a particular case of Lovelock theory), and $\mathcal{G}_{A B}^{(2)}$ is the Lanczos tensor $H_{A B}$ associated with the Gauss Bonnet Lagrangian.

For example, the Einstein Gauss Bonnet equations of motion up to $n=2$, without cosmological constant are:

$G_{B}^{A}+\gamma_{2} H_{B}^{A}=T_{B}^{A}$

where the Lanczos tensor is:

$$
\begin{aligned}
H_{A B}= & 2\left(R R_{A B}-2 R_{A C} R_{B}^{C}-2 R^{C D} R_{A C B D}\right. \\
& \left.+R_{A}^{C D E} R_{B C D E}\right)-\frac{1}{2} g_{A B} L_{2} .
\end{aligned}
$$

\subsection{Pure Lovelock case}

Pure Lovelock is a theory that involving only a single fixed value of $n$ (with $n \geq 1$ ), without sum over the lower order. In some cases it is considered one single value of $n \geq 1$ plus the $n=0$ term, i.e. $L=L_{0}+L_{n}$, as for example in Refs. $[3,34,35]$. For simplicity, in this work we take a single value of $L_{n}$ without the $L_{0}$ term, as for example in Refs. [37,41]. Thus, the Lagrangian is:

$L=\sqrt{-g} \gamma_{n} L_{n}=\sqrt{-g} \gamma_{n} \frac{1}{2^{n}} \delta_{\alpha_{1} \beta_{1} \cdots \alpha_{n} \beta_{n}}^{\mu_{1} v_{1} \cdots \mu_{n} v_{n}} \Pi_{r=1}^{n} R_{\mu_{r} v_{r}}^{\alpha_{r} \beta_{r}}$,

The equations of motion are given by:

$\mathcal{G}_{A B}^{(n)}=T_{A B}$,

where

$\left(\mathcal{G}^{(n)}\right)_{B}^{A}=-\frac{1}{2^{n+1}} \delta_{B A_{1} \cdots A_{2 n}}^{A B_{1} \cdots B_{2 n}} R_{B_{1} B_{2}}^{A_{1} A_{2}} \cdots R_{B_{2 n-1} B_{2 n}}^{A_{2 n-1} A_{2 n}}$.

and where the coupling constants were set to unity as in Refs. [37,41].

In this work we study the static $d$ dimensional spherically symmetric metric, wich in Schwarzschild-like coordinates reads:

$d s^{2}=-e^{\nu(r)}+e^{\lambda(r)} d r^{2}+r^{2} d \Omega_{d-2}^{2}$,

where $d \Omega_{d-2}^{2}$ corresponds to the metric of a $(d-2)$ unitary sphere. The energy momentum tensor corresponds to a neutral perfect fluid: 
$T_{B}^{A}=\operatorname{diag}\left(-\rho, p_{r}, p_{\theta}, p_{\theta}, \ldots\right)$,

where, from the spherical symmetry, we have for all the $(d-2)$ angular coordinates that $p_{\theta}=p_{\phi}=\cdots$. The conservation law $T_{; B}^{A B}=0$ gives:

$\frac{1}{2}\left(p_{r}+\rho\right) v^{\prime}+p_{r}^{\prime}+\frac{d-2}{r}\left(p_{r}-p_{\theta}\right)=0$.

Note that the analogue Einstein tensor has free divergence $\left(\mathcal{G}^{(n)}\right)_{B B}^{A B}=0$ [42], and the Bianchi Identities are satisfied [43].

It is worth stressing that the $(d-2)$ angular components of the equations of motion are similar $(\theta, \theta)=(\phi, \phi)=\cdots$ and the conservation equation can be written as a combination of the $(t, t),(r, r)$ and $(\theta, \theta)$ components [37]. In this way, there are three field equations $(t, t),(r, r),(\theta, \theta)=(\phi, \phi)=$ $\cdots$ and one conservation Eq. 14. But only three equations are independent. Thus, any one equation could be ignored and the system will be satisfied if the other three are solved.

\section{Pure Lovelock equations of motion for multiples sources}

In the equations of motion $10, T_{B}^{A}=\operatorname{diag}\left(-\rho, p_{r}, p_{\theta}, p_{\theta}\right.$, $\ldots)$ is given by Eq. 1, and the seed energy momentum tensor is given by $\bar{T}_{B}^{A}=\operatorname{diag}\left(-\bar{\rho}, \bar{p}_{r}, \bar{p}_{\theta}, \bar{p}_{\theta}, \cdots\right)$.

In the Gravitational Decoupling method, Ref. [5], developed for Einstein Hilbert theory, is introduced an additional source $\left(\theta_{1}\right)_{B}^{A}$ coupled with the seed energy momentum by the constant $\alpha$. It is worth to notice that the power of $\alpha$ coincides with the value $n=1$ corresponding to the Einstein Hilbert theory. Thus, the energy momentum 1 is:

$T_{B}^{A}=\bar{T}_{B}^{A}+\alpha\left(\theta_{1}\right)_{B}^{A}$

where the source $\left(\theta_{1}\right)_{B}^{A}$ is arbitrary. Thus, it is easily see that:

$$
\begin{aligned}
\rho & =\bar{\rho}-\alpha\left(\theta_{1}\right)_{0}^{0} \\
p_{r} & =\bar{p}_{r}+\alpha\left(\theta_{1}\right)_{1}^{1} \\
p_{\theta} & =\bar{p}_{\theta}+\alpha\left(\theta_{1}\right)_{2}^{2}
\end{aligned}
$$

In this work, inspired by the above mentioned method, for a generic value of $n$, it is proposed the following energy momentum tensor:

$$
\begin{aligned}
T_{B}^{A}= & \bar{T}_{B}^{A}+\alpha\left(\theta_{1}\right)_{B}^{A}+\alpha^{2}\left(\theta_{2}\right)_{B}^{A}+\cdots \\
& +\alpha^{n-1}\left(\theta_{n-1}\right)_{B}^{A}+\alpha^{n}\left(\theta_{n}\right)_{B}^{A}
\end{aligned}
$$

therefore the number of sources is determined by the value of $n$. It is worth to stress that the energy momentum 15 is a particular case of 19 for $n=1$. As example, for the Pure Gauss Bonnet case with $n=2$ the energy momentum 19 has the form $T_{B}^{A}=\bar{T}_{B}^{A}+\alpha\left(\theta_{1}\right)_{B}^{A}+\alpha^{2}\left(\theta_{2}\right)_{B}^{A}$. Now:

$$
\begin{aligned}
\rho= & \bar{\rho}-\alpha\left(\theta_{1}\right)_{0}^{0}-\alpha^{2}\left(\theta_{2}\right)_{0}^{0}-\cdots \\
& -\alpha^{n-1}\left(\theta_{n-1}\right)_{0}^{0}-\alpha^{n}\left(\theta_{n}\right)_{0}^{0} \\
p_{r}= & \bar{p}_{r}+\alpha\left(\theta_{1}\right)_{1}^{1}+\alpha^{2}\left(\theta_{2}\right)_{1}^{1}+\cdots \\
& +\alpha^{n-1}\left(\theta_{n-1}\right)_{1}^{1}+\alpha^{n}\left(\theta_{n}\right)_{1}^{1} \\
p_{\theta}= & \bar{p}_{\theta}+\alpha\left(\theta_{1}\right)_{2}^{2}+\alpha^{2}\left(\theta_{2}\right)_{2}^{2}+\cdots \\
& +\alpha^{n-1}\left(\theta_{n-1}\right)_{2}^{2}+\alpha^{n}\left(\theta_{n}\right)_{2}^{2}
\end{aligned}
$$

where $p_{\theta}=p_{\phi}=\cdots$. In Eq. $22 \bar{p}_{\theta}=\bar{p}_{\phi}=\cdots$ and we impose that $\left(\theta_{i}\right)_{2}^{2}=\left(\theta_{i}\right)_{3}^{3}=\cdots$.

So, for $\left(\theta_{i}\right)_{1}^{1} \neq\left(\theta_{i}\right)_{2}^{2}=\left(\theta_{i}\right)_{3}^{3}=\cdots$ and $\bar{p}_{r} \neq \bar{p}_{\theta}=$ $\bar{p}_{\phi}=\cdots$ these sources induce an anisotropy:

$$
\begin{aligned}
\Pi= & \bar{p}_{\theta}-\bar{p}_{r}+\alpha\left(\left(\theta_{1}\right)_{2}^{2}-\left(\theta_{1}\right)_{1}^{1}\right)+\alpha^{2}\left(\left(\theta_{2}\right)_{2}^{2}-\left(\theta_{2}\right)_{1}^{1}\right)+\cdots \\
& +\alpha^{n-1}\left(\left(\theta_{n-1}\right)_{2}^{2}-\left(\theta_{n-1}\right)_{1}^{1}\right) \\
& +\alpha^{n}\left(\left(\theta_{n}\right)_{2}^{2}-\left(\theta_{n}\right)_{1}^{1}\right) .
\end{aligned}
$$

For the isotropic case where $\bar{p}_{\theta}=\bar{p}_{r}$, the addition of our source $\theta_{A B}$ is a simple way to generate an anisotropy.

So, the $(t, t)$ and $(r, r)$ components of the equations of motion are given by $[37,41]$ :

$$
\begin{aligned}
& \frac{2}{d-2} r^{d-2}\left(\bar{\rho}-\alpha\left(\theta_{1}\right)_{0}^{0}-\alpha^{2}\left(\theta_{2}\right)_{0}^{0}-\cdots-\alpha^{n-1}\left(\theta_{n-1}\right)_{0}^{0}\right. \\
& \left.-\alpha^{n}\left(\theta_{n}\right)_{0}^{0}\right)=\frac{d}{d r}\left(r^{d-2 n-1}\left(1-e^{-\lambda}\right)^{n}\right),
\end{aligned}
$$

and

$$
\begin{aligned}
& \frac{2}{d-2} r^{2 n}\left(\bar{p}_{r}+\alpha\left(\theta_{1}\right)_{1}^{1}+\alpha^{2}\left(\theta_{2}\right)_{1}^{1}+\cdots+\alpha^{n-1}\left(\theta_{n-1}\right)_{1}^{1}\right. \\
& \left.\quad+\alpha^{n}\left(\theta_{n}\right)_{1}^{1}\right)=n r v^{\prime} e^{-\lambda}\left(1-e^{-\lambda}\right)^{n-1} \\
& \quad-(d-2 n-1)\left(1-e^{-\lambda}\right)^{n}
\end{aligned}
$$

We solve the $(t, t)$ and $(r, r)$ components of the Pure Lovelock equations together with the conservation equation. Using the Bianchi identities, we ignore the remaining $(\theta, \theta)=(\phi, \phi)=\cdots$ components (the suspense points indicate that all the tangential components of the Pure Lovelock equations are similar).

By inserting Eqs. 20, 21 and 22 into Eq. 14:

$$
\begin{aligned}
& \frac{1}{2}\left(\bar{p}_{r}+\bar{\rho}\right) v^{\prime}+\bar{p}_{r}^{\prime}+\frac{d-2}{r}\left(\bar{p}_{r}-\bar{p}_{\theta}\right) \\
& \quad+\alpha\left(\frac{1}{2}\left(\left(\theta_{1}\right)_{1}^{1}-\left(\theta_{1}\right)_{0}^{0}\right) v^{\prime}+\left(\left(\theta_{1}\right)_{1}^{1}\right)^{\prime}\right. \\
& \left.\quad+\frac{d-2}{r}\left(\left(\theta_{1}\right)_{1}^{1}-\left(\theta_{1}\right)_{2}^{2}\right)\right)
\end{aligned}
$$




$$
\begin{aligned}
& +\alpha^{2}\left(\frac{1}{2}\left(\left(\theta_{2}\right)_{1}^{1}-\left(\theta_{2}\right)_{0}^{0}\right) v^{\prime}+\left(\left(\theta_{2}\right)_{1}^{1}\right)^{\prime}\right. \\
& \left.+\frac{d-2}{r}\left(\left(\theta_{2}\right)_{1}^{1}-\left(\theta_{2}\right)_{2}^{2}\right)\right) \\
& +\cdots+\alpha^{n-1}\left(\frac{1}{2}\left(\left(\theta_{n-1}\right)_{1}^{1}-\left(\theta_{n-1}\right)_{0}^{0}\right) v^{\prime}+\left(\left(\theta_{n-1}\right)_{1}^{1}\right)^{\prime}\right. \\
& \left.+\frac{d-2}{r}\left(\left(\theta_{n-1}\right)_{1}^{1}-\left(\theta_{n-1}\right)_{2}^{2}\right)\right) \\
& +\alpha^{n}\left(\frac{1}{2}\left(\left(\theta_{n}\right)_{1}^{1}-\left(\theta_{n}\right)_{0}^{0}\right) v^{\prime}+\left(\left(\theta_{n}\right)_{1}^{1}\right)^{\prime}\right. \\
& \left.+\frac{d-2}{r}\left(\left(\theta_{n}\right)_{1}^{1}-\left(\theta_{n}\right)_{2}^{2}\right)\right) \\
& =0 .
\end{aligned}
$$

Thus, the system to solve corresponds to Eqs. 24, 25 and 26. At this stage we would deal with five unknown functions, namely, the metric components $\lambda(r)$ and $v(r)$, and the effective thermodynamics functions $\rho, p_{r}$ and $p_{\theta}=p_{\phi}=\cdots$. However, below, we implement the gravitational decoupling method where this scenario is modified.

The Pure Lovelock equations of motion for the seed energy momentum tensor are recovered for the limit $\alpha \rightarrow 0$ in the system 24, 25 and 26. So, it is fulfilled that

$\nabla_{A} \bar{T}_{B}^{A}=0$

and next, the first line of Eq. 26 is conserved. For $n=1$ both components of energy momentum tensor are directly conserved, 1.e $\nabla_{A}\left(\theta_{1}\right)_{B}^{A}=0$, however, for $n>1$ one can notice that:

$$
\begin{aligned}
& \alpha \nabla_{A}\left(\theta_{1}\right)_{B}^{A}+\alpha^{2} \nabla_{A}\left(\theta_{2}\right)_{B}^{A}+\cdots+\alpha^{n-1} \nabla_{A}\left(\theta_{n-1}\right)_{B}^{A} \\
& +\alpha^{n} \nabla_{A}\left(\theta_{n}\right)_{B}^{A}=0,
\end{aligned}
$$

where the covariant derivative is computed by using the line element 12 . In this work we impose in arbitrarily way that:

$\alpha^{i} \nabla_{A}\left(\theta_{i}\right)_{B}^{A}=0$

Thus, we will solve the system 24, 25, 27 and 29. Under this assumption each source is separately conserved, and thus, there is no exchange of energy momentum between them. Therefore, our energy momentum tensor 19 is a specific way of decoupling the system inspired by the approach of Ref. [5].

\section{Gravitational decoupling by MGD in Pure Lovelock gravity}

We start with a solution to Eqs. 24, 25, 27 and 29 with $\alpha=0$, namely seed solution $\left\{\eta, \mu, \bar{\rho}, \bar{p}_{r}, \bar{p}_{t}\right\}$, where $\eta$ and $\mu$ are the corresponding metric functions: $d s^{2}=-e^{\eta(r)} d t^{2}+\mu(r)^{-1} d r^{2}+r^{2} d \Omega_{d-2}^{2}$.

Turning on the parameter $\alpha$, the effects of the sources $\left(\theta_{i}\right)_{A B}$ appear on the seed solution $\left\{\eta, \mu, \bar{\rho}, \bar{p}_{r}, \bar{p}_{t}\right\}$. These effects can be encoded in the geometric deformation undergone by the seed fluid geometry $\{\eta, \mu\}$ in Eq. 30 as follows:

$\eta(r) \rightarrow v(r)=\eta(r)$

$\mu(r) \rightarrow e^{-\lambda}=\mu(r)-\alpha g(r)$.

It means that only the radial component of the line element 30 is deformed, where $g(r)$ is the corresponding deformation of the radial part. This is known as Minimal Geometric Deformation [5] .Thus, replacing Eqs. 31 and 32 into of Eqs. 24 and 25 :

$$
\begin{aligned}
& \frac{2}{d-2} r^{d-2}\left(\bar{\rho}-\alpha\left(\theta_{1}\right)_{0}^{0}-\alpha^{2}\left(\theta_{2}\right)_{0}^{0}-\cdots-\alpha^{n-1}\left(\theta_{n-1}\right)_{0}^{0}\right. \\
& \left.-\alpha^{n}\left(\theta_{n}\right)_{0}^{0}\right)=\frac{d}{d r}\left(r^{d-2 n-1}((1-\mu)+\alpha g)^{n}\right)
\end{aligned}
$$

and

$$
\begin{aligned}
& \frac{2}{d-2} r^{2 n}\left(\bar{p}_{r}+\alpha\left(\theta_{1}\right)_{1}^{1}+\alpha^{2}\left(\theta_{2}\right)_{1}^{1}+\cdots+\alpha^{n-1}\left(\theta_{n-1}\right)_{1}^{1}\right. \\
& \left.+\alpha^{n}\left(\theta_{n}\right)_{1}^{1}\right)=n r v^{\prime}(\mu-\alpha g)((1-\mu)+\alpha g)^{n-1} \\
& -(d-2 n-1)((1-\mu)+\alpha g)^{n}
\end{aligned}
$$

Thus we must solve the system of Eqs. 33, 34, 27 and 29. We use the Binomial theorem :

$$
\begin{aligned}
(x+y)^{N}= & x^{N}+N x^{N-1} y+\left(\begin{array}{c}
N \\
2
\end{array}\right) x^{N-2} y^{2}+\cdots \\
& +N x y^{N-1}+y^{N}
\end{aligned}
$$

thus:

$$
\begin{aligned}
((1-\mu)+\alpha g)^{n}= & (1-\mu)^{n}+n(1-\mu)^{n-1} g \alpha \\
& +\left(\begin{array}{c}
n \\
2
\end{array}\right)(1-\mu)^{n-2} g^{2} \alpha^{2}+\cdots \\
& +n(1-\mu) g^{n-1} \alpha^{n-1}+g^{n} \alpha^{n}
\end{aligned}
$$

and

$$
\begin{aligned}
((1-\mu)+\alpha g)^{n-1}= & (1-\mu)^{n-1}+(n-1)(1-\mu)^{n-2} g \alpha \\
& +\left(\begin{array}{c}
n-1 \\
2
\end{array}\right)(1-\mu)^{n-3} g^{2} \alpha^{2}+\cdots \\
& +(n-1)(1-\mu) g^{n-2} \alpha^{n-2}+g^{n-1} \alpha^{n-1},
\end{aligned}
$$

Thus, replacing Eqs. 36 and 37 into of Eqs. 33 and 34, the system splits into the following sets of equations: 
- The standard Pure Lovelock equations for a seed solution (with $\alpha=0$ ) :

$\frac{2}{d-2} r^{d-2} \bar{\rho}=\frac{d}{d r}\left(r^{d-2 n-1}(1-\mu)^{n}\right)$,

and

$$
\begin{aligned}
\frac{2}{d-2} r^{2 n}\left(\bar{p}_{r}\right)= & n r v^{\prime} \mu(1-\mu)^{n-1} \\
& -(d-2 n-1)(1-\mu)^{n}
\end{aligned}
$$

and the respective conservation equation:

$$
\frac{1}{2}\left(\bar{p}_{r}+\bar{\rho}\right) v^{\prime}+\bar{p}_{r}^{\prime}+\frac{d-2}{r}\left(\bar{p}_{r}-\bar{p}_{\theta}\right)=0
$$

- The terms of order $\alpha$ give rise to the following quasi-Pure Lovelock equations of order $\alpha^{1}$, which include the source $\theta_{A B}$ :

$$
\begin{gathered}
-\frac{2}{d-2} r^{d-2}\left(\theta_{1}\right)_{0}^{0}=\frac{d}{d r}\left(r^{d-2 n-1} n(1-\mu)^{n-1} g\right) \\
\frac{2}{d-2} r^{2 n}\left(\theta_{1}\right)_{1}^{1}=n(1-\mu)^{n-1} g\left(r v ^ { \prime } \left((n-1) \mu(1-\mu)^{-1}\right.\right. \\
-1)-(d-2 n-1))
\end{gathered}
$$

and the respective conservation equation:

$$
\frac{1}{2}\left(\left(\theta_{1}\right)_{1}^{1}-\left(\theta_{1}\right)_{0}^{0}\right) v^{\prime}+\left(\left(\theta_{1}\right)_{1}^{1}\right)^{\prime}+\frac{d-2}{r}\left(\left(\theta_{1}\right)_{1}^{1}-\left(\theta_{1}\right)_{2}^{2}\right)=0
$$

Thus, following the iteration, it is possible to obtain the quasi pure Lovelock equations of order $\alpha^{2}, \alpha^{3}, \ldots, \alpha^{n-3}$, $\alpha^{n-2}$.

- The terms of order $\alpha^{n-1}$ give rise to the following quasiPure Lovelock equations of order $\alpha^{n-1}$ :

$$
-\frac{2}{d-2} r^{d-2}\left(\theta_{n-1}\right)_{0}^{0}=\frac{d}{d r}\left(r^{d-2 n-1} n(1-\mu) g^{n-1}\right),
$$

and

$$
\begin{aligned}
\frac{2}{d-2} r^{2 n}\left(\theta_{n-1}\right)_{1}^{1}= & n g^{n-1}\left(r v^{\prime}(\mu-(n-1)(1-\mu))\right. \\
& -(d-2 n-1)(1-\mu))
\end{aligned}
$$

and the respective conservation equation

$$
\begin{aligned}
& \frac{1}{2}\left(\left(\theta_{n-1}\right)_{1}^{1}-\left(\theta_{n-1}\right)_{0}^{0}\right) v^{\prime}+\left(\left(\theta_{n-1}\right)_{1}^{1}\right)^{\prime} \\
& +\frac{d-2}{r}\left(\left(\theta_{n-1}\right)_{1}^{1}-\left(\theta_{n-1}\right)_{2}^{2}\right)=0 .
\end{aligned}
$$

- The terms of order $\alpha^{n}$ give rise to the following quasiPure Lovelock equations of order $\alpha^{n}$ :

$$
-\frac{2}{d-2} r^{d-2}\left(\theta_{n}\right)_{0}^{0}=\frac{d}{d r}\left(r^{d-2 n-1} g^{n}\right)
$$

and

$$
\frac{2}{d-2} r^{2 n}\left(\theta_{n}\right)_{1}^{1}=-g^{n}\left(n r v^{\prime}+(d-2 n-1)\right)
$$

and the respective conservation equation

$$
\frac{1}{2}\left(\left(\theta_{n}\right)_{1}^{1}-\left(\theta_{n}\right)_{0}^{0}\right) v^{\prime}+\left(\left(\theta_{n}\right)_{1}^{1}\right)^{\prime}+\frac{d-2}{r}\left(\left(\theta_{n}\right)_{1}^{1}-\left(\theta_{n}\right)_{2}^{2}\right)=0 .
$$

It is worth stressing that each quasi Pure Lovelock equation cannot be formally identified as the spherically symmetric Pure Lovelock equations for $n>1$, because the right sides of each quasi Pure Lovelock equation do not have the standard expressions for the Generalized Einstein tensor components $\mathcal{G}_{00}^{(n)}$ and $\mathcal{G}_{11}^{(n)}$. Furthermore, the Bianchi identities are not satisfied for each quasi Pure Lovelock equation. For $n=1$ the quasi Einstein equations can be transformed in the standard Einstein equations after a convenient redefinition of the energy momentum tensor [5], however, the method of the Ref. [5] has been widely used to find new solutions without using this mentioned redefinition in several works.

Despite the above mentioned, our imposed way for solving the system 33, 34, 27 and 29, based in the decoupling of sources by means of the standard and quasi Pure Lovelock equations, ensures us to solve successfully the original system 33, 34 and 26. Furthermore, under our assumptions, each conservation Eqs. 40, 43, ․ 46, 49 is separately conserved, and thus, there is no exchange of energy momentum between the seed fluid and each sector $\left(\theta_{i}\right)_{A B}$. So, in our gravitational decoupling method there is only purely gravitational interaction.

It is worth stressing that as a consequence of the application of the MGD:

- We start with the indefinite system 24, 25 and 26. After the application of MGD, we have a set of equations for the seed fluid ( $\left.\nu, \mu, \bar{\rho}, \bar{p}_{r}, \bar{p}_{\theta}\right)$ given by the standard Pure Lovelock equations.

Next, we suppose that we have already found a seed fluid solution $(v, \mu)$ and the sources $\left(\bar{\rho}, \bar{p}_{r}, \bar{p}_{\theta}\right)$, thus we have :

- A much simpler system of four unknown functions $\left(g,\left(\theta_{n}\right)_{0}^{0},\left(\theta_{n}\right)_{1}^{1},\left(\theta_{n}\right)_{2}^{2}\right)$ given by the quasi Pure Lovelock equations of order $\alpha^{n}$.

- Supposing that we have found the values of $g$ and $\left(\theta_{n}\right)_{A B}$, we have $n-1$ systems, given by the quasi Pure Love- 
lock equations of order $\alpha^{i}$, where each of them has three unknown functions $\left(\left(\theta_{i}\right)_{0}^{0},\left(\theta_{i}\right)_{1}^{1},\left(\theta_{i}\right)_{2}^{2}\right)$

For the study of well behaved solutions that represent stellar distributions it is necessary to analyse the matching conditions [13]. This is outside of the scope of this work and could be studied in elsewhere.

\section{A special case}

We impose the condition $v=-\lambda$ in Eq. 12. So, the $(t, t)$ component of Pure Lovelock equation keeps its form as in Eq. 24 . However, it is direct to check that the $(r, r)$ component, Eq. 25, now is:

$$
\begin{aligned}
& \frac{2}{d-2} r^{d-2}\left(-\bar{p}_{r}-\alpha\left(\theta_{1}\right)_{1}^{1}-\alpha^{2}\left(\theta_{2}\right)_{1}^{1}-\cdots-\alpha^{n-1}\left(\theta_{n-1}\right)_{1}^{1}\right. \\
& \left.-\alpha^{n}\left(\theta_{n}\right)_{1}^{1}\right)=\frac{d}{d r}\left(r^{d-2 n-1}\left(1-e^{-\lambda}\right)^{n}\right)
\end{aligned}
$$

see Ref. [32]. Now, the system to solve is: Eq. 24 that corresponds to $(t, t)$ component, Eq. 50 that corresponds to $(r, r)$ component, and Eqs. 27 and 29.

Furthermore, we impose the condition $\rho=-p_{r}$, as in Refs. [3,44], where $\rho$ corresponds to Eq. 20 and $p_{r}$ to Eq. 21. Additionally, we will impose arbitrarily that $\bar{\rho}=-\bar{p}_{r}$ and $\left(\theta_{i}\right)_{0}^{0}=\left(\theta_{i}\right)_{1}^{1}$, with $i=1,2, \ldots, n$. Thus, the condition $\rho=-p_{r}$ is fulfilled.

So, the $(t, t)$ and $(r, r)$ components are similar to Eq. 24, whereas, now the conservation Eq. 26 takes the following form:

$$
\begin{aligned}
& \bar{p}_{r}^{\prime}+\frac{d-2}{r}\left(\bar{p}_{r}-\bar{p}_{t}\right) \\
& +\alpha\left(\left(\left(\theta_{1}\right)_{1}^{1}\right)^{\prime}+\frac{d-2}{r}\left(\left(\theta_{1}\right)_{1}^{1}-\left(\theta_{1}\right)_{2}^{2}\right)\right) \\
& +\alpha^{2}\left(\left(\left(\theta_{2}\right)_{1}^{1}\right)^{\prime}+\frac{d-2}{r}\left(\left(\theta_{2}\right)_{1}^{1}-\left(\theta_{2}\right)_{2}^{2}\right)\right)+\cdots \\
& +\alpha^{n-1}\left(\left(\left(\theta_{n-1}\right)_{1}^{1}\right)^{\prime}+\frac{d-2}{r}\left(\left(\theta_{n-1}\right)_{1}^{1}-\left(\theta_{n-1}\right)_{2}^{2}\right)\right) \\
& +\alpha^{n}\left(\left(\left(\theta_{n}\right)_{1}^{1}\right)^{\prime}+\frac{d-2}{r}\left(\left(\theta_{n}\right)_{1}^{1}-\left(\theta_{n}\right)_{2}^{2}\right)\right)=0
\end{aligned}
$$

In this way, the system to solve corresponds to the Eqs. 24, 27 with $\bar{\rho}=-\bar{p}_{r}$ and 29 with $\left(\theta_{i}\right)_{0}^{0}=\left(\theta_{i}\right)_{1}^{1}$. Our seed solution, which is solution of this system with $\alpha=0$, is:

$d s^{2}=-\mu(r) d t^{2}+\mu(r)^{-1} d r^{2}+r^{2} d \Omega_{d-2}^{2}$.

Again, turning on $\alpha$, the effects of the source $\theta_{A B}$ appear on the seed solution. These effects are encoded in the geometric deformation undergone by the seed fluid geometry in Eq. 52 as follows:

$$
\mu(r) \rightarrow e^{v}=\mu(r)-\alpha g(r) .
$$$$
\mu(r) \rightarrow e^{-\lambda}=\mu(r)-\alpha g(r) . .
$$

Thus, taking into account the geometric deformation of Eqs. 53 and 54, the $(t, t)$ and $(r, r)$ components are similar to the Eq. 33 and, the system to solve is given by Eqs. 33, 27 with $\bar{\rho}=-\bar{p}_{r}$ and 29 with $\left(\theta_{i}\right)_{0}^{0}=\left(\theta_{i}\right)_{1}^{1}$.

Finally, using the binomial development 36, the system splits into the following sets of equations:

- The standard Pure Lovelock equations for a seed solution (with $\alpha=0$ ), that correspond to the Eqs. 38 and the conservation equation given by:

$$
\bar{p}_{r}^{\prime}+\frac{d-2}{r}\left(\bar{p}_{r}-\bar{p}_{\theta}\right)=0
$$

- The terms of order $\alpha$ give rise to the quasi-Pure Lovelock equations of order $\alpha^{1}$, that correspond to the Eqs. 41 and the conservation equation given by:

$$
\left(\left(\theta_{1}\right)_{1}^{1}\right)^{\prime}+\frac{d-2}{r}\left(\left(\theta_{1}\right)_{1}^{1}-\left(\theta_{1}\right)_{2}^{2}\right)=0
$$

Again, following the iteration, it is possible to obtain the quasi pure Lovelock equations of order $\alpha^{2}, \alpha^{3}, \ldots, \alpha^{n-3}$, $\alpha^{n-2}$.

- The terms of order $\alpha^{n-1}$ give rise to the quasi-Pure Lovelock equations of order $\alpha^{n-1}$, that correspond to the Eq. 44 and the conservation equation given by:

$$
\left(\left(\theta_{n-1}\right)_{1}^{1}\right)^{\prime}+\frac{d-2}{r}\left(\left(\theta_{n-1}\right)_{1}^{1}-\left(\theta_{n-1}\right)_{2}^{2}\right)=0
$$

- The terms of order $\alpha^{n}$ give rise to the quasi-Pure Lovelock equations of order $\alpha^{n}$, that correspond to the Eqs. 47 and the conservation equation given by:

$$
\left(\left(\theta_{n}\right)_{1}^{1}\right)^{\prime}+\frac{d-2}{r}\left(\left(\theta_{n}\right)_{1}^{1}-\left(\theta_{n}\right)_{2}^{2}\right)=0 .
$$

\subsection{A very simple test: applying the method to the AdS} space time

As a simple test, we apply our method to a seed Anti de Sitter space time and, we test if it is possible to obtain a solution of physical interest that represents regular black holes.

The Schwarzschild black hole has a singularity where the laws of physics cease to operate. From the classical point of view, Bardeen in Ref. [45] proposed the first model of regular black holes, where the singularity is avoided, due to the formation of a dense core near the origin, whose effective cosmological constant $\Lambda_{\text {eff }}$ causes repulsive effects and, whose internal geometry has de Sitter form. This is achieved, by the change of the constant mass parameter by a mass function 
$M \rightarrow m(r)$, such that near the origin $m(r) \approx \frac{\Lambda_{\text {eff }}}{6} r^{3}$, and $\lim _{r \rightarrow \infty} m(r)=M$. Thus, the factor $f=1-\frac{2 m(r)}{r}$ near the origin behaves as de Sitter space time, and far from origin behaves as Scharzschild space time. After this, the formation of the Sitter core is associated to quantum fluctuations, where the energy density is of order of Planck units near the origin. This model is called Planck star, see Refs. [46,47]. Other more recent studies about regular black holes have been developed in Refs. [3,48-51].

Due that regular black holes with de Sitter ground state have a cosmological horizon, we apply the method to a seed solution with Anti de Sitter structure. This is because, the presence of the cosmological horizon prevents a correct definition of the mass. Conversely, Anti de Sitter space time has a well defined asymptotically region, and thus, it is possible to define the mass.

As was said above, Pure Lovelock is a theory that, for $\Lambda>0$, has a unique de Sitter ground state for $n$ odd, and has a double Anti de Sitter or de Sitter ground state for $n$ even. Conversely, for $\Lambda<0$, has a unique Anti de Sitter ground state for $n$ odd and does not have physical solution for $n$ even.

So, to find a seed solution with Anti de Sitter structure, we study the case where the seed fluid has the following energy density:

$\bar{\rho}=-\bar{p}_{r}=\Lambda_{d}=\frac{(d-1)(d-2)}{2 l^{2 n}}$, for $n$ even,

and

$\bar{\rho}=-\bar{p}_{r}=\Lambda_{d}=-\frac{(d-1)(d-2)}{2 l^{2 n}}$, for $n$ odd,

thus, the energy density represents a $d$ dimensional cosmological constant, and $l^{n}$ is the Anti de Sitter radius.

Then, we will apply the Gravitational Decoupling method as follows:

- We solve the standard Pure Lovelock equations for our seed fluid:

First, we test the case with $n$ even. Inserting the seed energy density 59 into Eq. 38, we get to

$\frac{r^{2 n}}{l^{2 n}}=(1-\mu)^{n}$

where $d-2 n-1>0$ and,whose solution is $\pm \frac{r^{2}}{l^{2}}=$ $(1-\mu)$. Taking the minus sign:

$\mu=1+\frac{r^{2}}{l^{2}}$

For the case with $n$ odd, with $d-2 n-1>0$, inserting the seed energy density 60 into Eq. 38 , it is direct that the unique solution is Eq. 62.
Thus, both for $n$ odd or $n$ even, our seed solutions represent an Anti de Sitter space time. The tangential seed pressure is determined by Eq. 55 .

- We solve the quasi-Pure Lovelock of order $\alpha^{n}$ :

We choose a source $-\left(\theta_{n}\right)_{0}^{0}=F(r)$ as in Ref. [3]: $F(r)$ has a maximum value at $r=0$, such that the $m(r)$ function near the origin behaves as :

$\left.m(r)\right|_{r \approx 0} \approx \frac{r^{d-1}}{k^{2 n}}$,

where $k$ is a constant, and the $m(r)$ function is computed as:

$m(r)=\frac{2}{d-2} \int_{0}^{r} F(r) r^{d-2} d r$

By solving Eq. 47:

$g(r)=\left(\frac{m(r)}{r^{d-2 n-1}}\right)^{1 / n}$

So, our line element is given by

$d s^{2}=-f(r) d t^{2}+\frac{d r^{2}}{f(r)}+r^{2} d \Omega_{d-2}^{2}$,

where:

$f(r)=\mu-\alpha g=1+\frac{r^{2}}{l^{2}}-\alpha\left(\frac{m(r)}{r^{d-2 n-1}}\right)^{1 / n}$,

thus, near the origin the function behaves as:

$\left.f(r)\right|_{r \approx 0}=1-\left(\frac{\alpha}{k^{2}}-\frac{1}{l^{2}}\right) r^{2}$,

thus, for

$\alpha>\frac{k^{2}}{l^{2}}$

the solution behaves as de Sitter near the origin, and represents to a Pure Lovelock regular black hole by gravitational decoupling.

One example of $F(r)$ function is found in Ref. [3], wich is a $d$ dimensional generalization of Hayward density and, in our case is:

$F(r)=\frac{(d-1)(d-2)}{2} \frac{Q^{d-2} M^{2}}{\left(Q^{d-2}+r^{d-1}\right)^{2}}$

which yields $m(r)=\frac{M r^{d-1}}{Q^{2} M+r^{d-1}}$, 
where, one can notice that:

$$
\begin{aligned}
& F(r)_{\max }=F(0)=\frac{(d-1)(d-2)}{2 Q^{d-2}} \\
& \text { and }\left.m(r)\right|_{r \approx 0} \approx \frac{1}{Q^{d-2}} r^{d-1},
\end{aligned}
$$

on the other hand:

$$
\lim _{r \rightarrow \infty} F(r)=0 \text { and } \lim _{r \rightarrow \infty} m(r)=M
$$

where $Q$ is defined in this reference as a regulator and $M$ is the total mass. There is a deep analysis of unities in Ref. [3]. So, $k^{2 n}=Q^{d-2}$ and if the condition 69 is satisfied, then the function $F(r)$ is suitable for represent a Pure Lovelock Regular black hole. The fact that $\lim _{r \rightarrow \infty} m(r)=$ $M$ allows to define correctly the mass in a space time with AdS ground state.

The source $\left(\theta_{n}\right)_{2}^{2}$ is determined with Eq. 58

- Known $\bar{\rho}, \bar{p}_{r}, \bar{p}_{t},\left(\theta_{n}\right)_{0}^{0},\left(\theta_{n}\right)_{1}^{1},\left(\theta_{n}\right)_{2}^{2}, \mu, v$ and $g$, the remaining sources $\left(\theta_{i}\right)_{0}^{0},\left(\theta_{i}\right)_{1}^{1},\left(\theta_{i}\right)_{2}^{2}$ are determined by the quasi-Pure Lovelock equations of order $\alpha^{i}$.

\section{Conclusion and discussion}

We have shown an approach that represents a simple method for decoupling gravitational sources in Pure Lovelock gravity. Thus, it is possible to decouple gravitational sources under the effects of higher curvature correction terms for space times with a number of dimensions greater than four.

Applying our method, the final solution obtained is the result of the decoupling of the Pure Lovelock equations in a seed sector described by the seed energy momentum tensor $\bar{T}_{B}^{A}$ and the quasi Pure Lovelock equations of order $\alpha^{i}$ described by the sources $\left(\theta_{i}\right)_{B}^{A}$. Thus, the equations of motion are solved for each sector separately and, by the superposition of these solutions, the complete solution is obtained.

The seed and the extra sources are separately conserved under the assumptions imposed in this work. The quasi Pure Lovelock equations are of order $\alpha^{1}, \alpha^{2}, \ldots, \alpha^{n-1}, \alpha^{n}$. Therefore, the combination of these $n+1$ sectors only has gravitational interaction and does not have exchange of energy momentum. The order $\alpha^{n}$ corresponds to the order of the Generalized Einstein Tensor $\mathcal{G}_{A B}^{(n)}$. Thus, for $n=1$, where $\mathcal{G}_{A B}^{(1)}$ represents to the Einstein Tensor in Einstein Hilbert theory, the quasi Pure Lovelock are of order $\alpha^{1}$, and then, the quasi Einstein equations described in Ref. [5] are a particular case of our method. As indicates Ref. [5], the quasi Einstein equations are useful to study the interaction between ordinary matter and the conjectured dark matter, so, our quasi Pure Lovelock equations perhaps could serve to investigate this problem in presence of higher curvature terms and in space times with a number of dimensions greater than four.
Furthermore, we have presented our method for the case where the line element has the form of Eq. 12 in Sect. 4, and for the case where $v=-\lambda$ and $\rho=-p_{r}$ in Sect. 5 . As a simple test, we have applied our method to an Anti de Sitter seed solution. Choosing a source $F(r)$ that fulfills the condition 63, we have found the Pure Lovelock Regular black hole solution by gravitational decoupling 67 . This solution differs in its structure with the Pure Lovelock regular black hole found in Ref. [3], whose structure is:

$f(r)=1-\left(\frac{m(r)}{r^{d-2 n-1}} \pm \frac{r^{2 n}}{l^{2 n}}\right)^{1 / n}$

wich takes the sign $+(-)$ for $\Lambda>(<) 0$. Thus, Eq. 67 is a new solution in Pure Lovelock gravity, and thus a new Pure Lovelock regular black hole. In our solution, the ground state is obtained with the seed fluid, and when the extra sources $\left(\theta_{i}\right)_{A B}$ are turn off. Although both solutions 67 and 73 differ in its structure, both share the same structure of ground state : for $\Lambda>0$ both solutions have a double Anti de Sitter or de Sitter ground state for $n$ even (where we have chosen the AdS branch), whereas for $\Lambda<0$ both solutions have a single Anti de Sitter ground state for $n$ odd.

Although our solution 67 is new by using Pure Lovelock gravity, one solution with similar mathematical structure was found by using the $n$-fold degenerated ground state theory in Ref. [3]. Therefore both solutions share the horizons structure and thermodynamics features described in Ref. [3]. However, both solutions have different ground state structure. The ground state in the $n$-fold degenerated ground state theory is obtained by choosing a suited election of the coupled constants $\gamma$ such that the solution has one unique Anti de Sitter ground state (or $n$ fold degenerated Anti de Sitter ground state) with $\Lambda<0$, whereas in our case the ground state structure is different and was described in the last paragraph.

So, we have showed that our Gravitational Decoupling method is a direct way to obtain regular black holes. In regarding this, the Minimal Geometric Deformation of the Anti de Sitter space time give rise to regular black holes, under the assumptions used in this work.

A simple recipe to apply our method could be:

- Pick up a seed Pure Lovelock solution $\left\{\mu, v, \bar{\rho}, \bar{p}_{r}, \bar{p}_{t}\right\}$ and solve the standard Pure Lovelock equations.

- Solve the quasi Pure Lovelock equations of order $\alpha^{n}$. In regarding this, we impose a form of the source $\left(\theta_{n}\right)_{0}^{0}$ and the function $g(r)$ is directly determined by the $(t, t)$ component. Furthermore, in direct way, the sources $\left(\theta_{n}\right)_{1}^{1}$ and $\left(\theta_{n}\right)_{2}^{2}$ are obtained by solving the $(r, r)$ and $(\theta, \theta)$ components, respectively.

- Well known $\bar{\rho}, \bar{p}_{r}, \bar{p}_{t},\left(\theta_{n}\right)_{0}^{0},\left(\theta_{n}\right)_{1}^{1},\left(\theta_{n}\right)_{2}^{2}, \mu, v$ and $g$, the remaining functions $\left(\theta_{i}\right)_{0}^{0},\left(\theta_{i}\right)_{1}^{1},\left(\theta_{i}\right)_{2}^{2}$ are directly determined by the quasi-Pure Lovelock equations of order $\alpha^{i}$. 
Thus, our method is an easy algorithm to search new analytical solutions of physical interest in Pure Lovelock gravity. In Einstein Hilbert theory, the Gravitational Decoupling method of Ref. [5] have been used to find new $4 D$ black hole solutions in Refs. [14-18] and new $4 D$ well behaved solutions that represent stellar distributions in Refs. [6-13,27]. So, inspired by this method, we have presented a useful tool that could serve to find new black hole solutions or stellar distributions in space times with a number of dimensions greater than four, and in presence of higher curvature correction terms. This applications could be studied in elsewhere.

Data Availability Statement This manuscript has no associated data or the data will not be deposited. [Authors' comment: This is a theoretical work and no experimental data were used.]

Open Access This article is distributed under the terms of the Creative Commons Attribution 4.0 International License (http://creativecomm ons.org/licenses/by/4.0/), which permits unrestricted use, distribution, and reproduction in any medium, provided you give appropriate credit to the original author(s) and the source, provide a link to the Creative Commons license, and indicate if changes were made.

Funded by SCOAP ${ }^{3}$.

\section{References}

1. D. Lovelock, The Einstein tensor and its generalizations. J. Math. Phys. 12, 498-501 (1971). https://doi.org/10.1063/1.1665613

2. N. Dadhich, A distinguishing gravitational property for gravitational equation in higher dimensions. Eur. Phys. J. C 76(3), 104 (2016). https://doi.org/10.1140/epjc/s10052-016-3933-z. arXiv:1506.08764

3. R. Aros, M. Estrada, Regular black holes and its thermodynamics in Lovelock gravity. Eur. Phys. J. C 79(3), 259 (2019). https://doi. org/10.1140/epjc/s10052-019-6783-7. arXiv:1901.08724

4. R.-G. Cai, N. Ohta, Black holes in pure lovelock gravities. Phys. Rev. D 74, 064001 (2006). https://doi.org/10.1103/PhysRevD.74. 064001. arXiv:hep-th/0604088

5. J. Ovalle, Decoupling gravitational sources in general relativity: from perfect to anisotropic fluids. Phys. Rev. D 95(10), 104019 (2017). https://doi.org/10.1103/PhysRevD.95. 104019. arXiv: 1704.05899

6. J. Ovalle, R. Casadio, R. da Rocha, A. Sotomayor, Anisotropic solutions by gravitational decoupling. Eur. Phys. J. C 78(2), 122 (2018). https://doi.org/10.1140/epjc/s10052-018-5606-6. arXiv: 1708.00407

7. C.L. Heras, P. Leon, Using MGD gravitational decoupling to extend the isotropic solutions of Einstein equations to the anisotropical domain. Fortsch. Phys. 66(7), 1800036 (2018). https://doi.org/10. 1002/prop.201800036. arXiv: 1804.06874

8. E. Morales, F. Tello-Ortiz, Charged anisotropic compact objects by gravitational decoupling. Eur. Phys. J. C 78(8), 618 (2018). https:// doi.org/10.1140/epjc/s10052-018-6102-8. arXiv:1805.00592

9. R. Pérez Graterol, A new anisotropic solution by MGD gravitational decoupling. Eur. Phys. J. Plus 133(6), 244 (2018). https:// doi.org/10.1140/epjp/i2018-12074-2

10. E. Morales, F. Tello-Ortiz, Compact anisotropic models in general relativity by gravitational decoupling. Eur. Phys. J. C 78(10), 841 (2018). https://doi.org/10.1140/epjc/s10052-018-6319-6. arXiv:1808.01699
11. S.K. Maurya, F. Tello-Ortiz, Generalized relativistic anisotropic compact star models by gravitational decoupling. Eur. Phys. J. C 79(1), 85 (2019). https://doi.org/10.1140/epjc/ s10052-019-6602-1

12. L. Gabbanelli, A. Rincon, C. Rubio, Gravitational decoupled anisotropies in compact stars. Eur. Phys. J. C 78(5), 370 (2018). https://doi.org/10.1140/epjc/s10052-018-5865-2. arXiv: 1802.08000

13. M. Estrada, F. Tello-Ortiz, A new family of analytical anisotropic solutions by gravitational decoupling. Eur. Phys. J. Plus 133(11), 453 (2018). https://doi.org/10.1140/epjp/i2018-12249-9. arXiv: 1803.02344

14. J. Ovalle, R. Casadio, R d Rocha, A. Sotomayor, Z. Stuchlik, Black holes by gravitational decoupling. Eur. Phys. J. C78(11), 960 (2018). https://doi.org/10.1140/epjc/s10052-018-6450-4. arXiv: 1804.03468

15. E. Contreras, P. Bargueño, Minimal geometric deformation decoupling in $2+1$ dimensional space-times. Eur. Phys. J. C 78(7), 558 (2018). https://doi.org/10.1140/epjc/ s10052-018-6048-x. arXiv:1805.10565

16. E. Contreras, P. Bargueño, Minimal geometric deformation in asymptotically (A-)dS space-times and the isotropic sector for a polytropic black hole. Eur. Phys. J. C 78(12), 985 (2018). https:// doi.org/10.1140/epjc/s10052-018-6472-y. arXiv:1809.09820

17. E. Contreras, Gravitational decoupling in $2+1$ dimensional space-times with cosmological term. Class. Quant. Grav. 36(9), 095004 (2019). https://doi.org/10.1088/1361-6382/ab11e6. arXiv: 1901.00231

18. E. Contreras, A. Rincon, P. Bargueno, A general interior anisotropic solution for a BTZ vacuum in the context of the minimal geometric deformation decoupling approach. Eur. Phys. J. C 79(3), 216 (2019). https://doi.org/10.1140/epjc/ s10052-019-6749-9. arXiv:1902.02033

19. J. Ovalle, R. Casadio, R. da Rocha, A. Sotomayor, Z. Stuchlik, Einstein-Klein-Gordon system by gravitational decoupling. EPL 124(2), 20004 (2018). https://doi.org/10.1209/0295-5075/ 124/20004. arXiv:1811.08559

20. M. Sharif, S. Saba, Gravitational decoupled anisotropic solutions in $f(\mathcal{G})$ gravity. Eur. Phys. J. C 78(11), 921 (2018). https://doi.org/ 10.1140/epjc/s10052-018-6406-8. arXiv:1811.08112

21. M. Sharif, A. Waseem, Anisotropic spherical solutions by gravitational decoupling in gravity. Ann. Phys. 405, 14-28 (2019). https:// doi.org/10.1016/j.aop.2019.03.003

22. G. Panotopoulos, A. Rincon, Minimal geometric deformation in a cloud of strings. Eur. Phys. J. C 78(10), 851 (2018). https://doi.org/ 10.1140/epjc/s10052-018-6321-z. arXiv:1810.08830

23. J. Ovalle, Decoupling gravitational sources in general relativity: the extended case. Phys. Lett. B 788, 213-218 (2019). https://doi. org/10.1016/j.physletb.2018.11.029. arXiv:1812.03000

24. E. Contreras, Minimal geometric deformation: the inverse problem. Eur. Phys. J. C 78(8), 678 (2018). https://doi.org/10.1140/epjc/ s10052-018-6168-3. arXiv:1807.03252

25. M. Sharif, S. Sadiq, Gravitational decoupled anisotropic solutions for cylindrical geometry. Eur. Phys. J. Plus 133(6), 245 (2018). https://doi.org/10.1140/epjp/i2018-12075-1

26. M. Sharif, S. Sadiq, Gravitational decoupled charged anisotropic spherical solutions. Eur. Phys. J. C 78(5), 410 (2018). https://doi. org/10.1140/epjc/s10052-018-5894-x. arXiv:1804.09616

27. L. Gabbanelli, J. Ovalle, A. Sotomayor, Z. Stuchlik, R. Casadio, A causal Schwarzschild-de Sitter interior solution by gravitational decoupling. Eur. Phys. J. C 79(6), 486 (2019). https://doi.org/10. 1140/epjc/s10052-019-7022-y. arXiv:1905.10162

28. M. Sharif, S. Sadiq, $2+1$-dimensional gravitational decoupled anisotropic solutions. Chin. J. Phys. 60, 279-289 (2019). https:// doi.org/10.1016/j.cjph.2019.05.018 
29. M. Estrada, R. Prado, The Gravitational decoupling method: the higher dimensional case to find new analytic solutions. Eur. Phys. J. Plus 134(4), 168 (2019). https://doi.org/10.1140/epjp/ i2019-12555-8. arXiv:1809.03591

30. J. Ovalle, C. Posada, Z. Stuchlík, Anisotropic ultracompact Schwarzschild star by gravitational decoupling. Class. Quant. Grav. 36(20), 205010 (2019). https://doi.org/10.1088/1361-6382/ ab4461. arXiv:1905.12452

31. R. Casadio, E. Contreras, J. Ovalle, A. Sotomayor, Z. Stuchlick, Isotropization and change of complexity by gravitational decoupling. Eur. Phys. J. C 79(10), 826 (2019). https://doi.org/10.1140/ epjc/s10052-019-7358-3. arXiv:1909.01902

32. N. Dadhich, On Lovelock vacuum solution. Math. Today 26, 37 (2011). arXiv:1006.0337

33. N. Dadhich, J.M. Pons, Static pure Lovelock black hole solutions with horizon topology $\mathrm{S}^{(n)} \times \mathrm{S}^{(n)}$. JHEP 05, 067 (2015). https:// doi.org/10.1007/JHEP05(2015)067. arXiv:1503.00974

34. J.M. Toledo, V.B. Bezerra, Black holes with quintessence in pure Lovelock gravity. Gen. Rel. Grav. 51(3), 41 (2019). https://doi.org/ 10.1007/s10714-019-2528-z

35. J.M. Toledo, V.B. Bezerra, Black holes with a cloud of strings in pure Lovelock gravity. Eur. Phys. J. C 79(2), 117 (2019). https:// doi.org/10.1140/epjc/s10052-019-6628-4

36. L. Aránguiz, X.-M. Kuang, O. Miskovic, Topological black holes in pure Gauss-Bonnet gravity and phase transitions. Phys. Rev. D 93(6), 064039 (2016). https://doi.org/10.1103/PhysRevD.93. 064039. arXiv: 1507.02309

37. N. Dadhich, S. Hansraj, B. Chilambwe, Compact objects in pure Lovelock theory. Int. J. Mod. Phys. D 26(06), 1750056 (2016). https://doi.org/10.1142/S0218271817500560. arXiv:1607.07095

38. N. Dadhich, S. Chakraborty, Buchdahl compactness limit for a pure Lovelock static fluid star. Phys. Rev. D 95(6), 064059 (2017). https://doi.org/10.1103/PhysRevD.95.064059. arXiv:1606.01330

39. A. Molina, N. Dadhich, A. Khugaev, Buchdahl-Vaidya-Tikekar model for stellar interior in pure Lovelock gravity. Gen. Rel. Grav. 49(7), 96 (2017). https://doi.org/10.1007/s10714-017-2259-y. arXiv: 1607.06229

40. R. Aros, M. Estrada, Regular black holes with $\Lambda>0$ and its evolution in Lovelock gravity. Eur. Phys. J. C 79(10), 810 (2019). https:// doi.org/10.1140/epjc/s10052-019-7316-0. arXiv:1906.01152
41. N. Dadhich, S. Hansraj, S.D. Maharaj, Universality of isothermal fluid spheres in Lovelock gravity. Phys. Rev. D 93(4), 044072 (2016). https://doi.org/10.1103/PhysRevD.93.044072. arXiv: 1510.07490

42. N. Dadhich, Characterization of the Lovelock gravity by Bianchi derivative. Pramana 74, 875-882 (2010). https://doi.org/10.1007/ s12043-010-0080-1. arXiv:0802.3034

43. X.O. Camanho, N. Dadhich, On Lovelock analogs of the Riemann tensor. Eur. Phys. J. C 76(3), 149 (2016). https://doi.org/10.1140/ epjc/s10052-016-3891-5. arXiv:1503.02889

44. I. Dymnikova, M. Korpusik, Regular black hole remnants in de Sitter space. Phys. Lett. B 685, 12-18 (2010). https://doi.org/10. 1016/j.physletb.2010.01.044

45. Bardeen, J.: Non-singular general-relativistic gravitacional collapse, Proceedings of the International Conference GR5, Tbilisi USSR

46. T. De Lorenzo, C. Pacilio, C. Rovelli, S. Speziale, On the effective metric of a Planck Star. Gen. Rel. Grav. 47(4), 41 (2015). https:// doi.org/10.1007/s10714-015-1882-8. arXiv:1412.6015

47. C. Rovelli, F. Vidotto, Planck stars. Int. J. Mod. Phys. D 23(12), 1442026 (2014). https://doi.org/10.1142/S0218271814420267. arXiv: 1401.6562

48. M.S. Ali, S.G. Ghosh, Thermodynamics of rotating Bardeen black holes: phase transitions and thermodynamics volume. Phys. Rev. D 99(2), 024015 (2019). https://doi.org/10.1103/PhysRevD.99. 024015

49. A.G. Tzikas, Bardeen black hole chemistry. Phys. Lett. B $\mathbf{7 8 8}$, 219-224 (2019). https://doi.org/10.1016/j.physletb.2018.11.036. arXiv: 1811.01104

50. A. Kumar, D. Veer Singh, S .G. Ghosh, D-dimensional Bardeen-AdS black holes in Einstein-Gauss-Bonnet theory. Eur. Phys. J. C79(3), 275 (2019). https://doi.org/10.1140/epjc/ s10052-019-6773-9. arXiv: 1808.06498

51. P.A. Cano, S. Chimento, T. Ortín, A. Ruipérez, Regular stringy black holes? Phys. Rev. D 99(4), 046014 (2019). https://doi.org/ 10.1103/PhysRevD.99.046014. arXiv:1806.08377 